\title{
Phase-matched coherent hard x-rays from relativistic high-order harmonic generation
}

\author{
Markus C. Kohler, Michael Klaiber, Karen Z. Hatsagortsyan ${ }^{*}$ and Christoph H. Keitel \\ Max-Planck-Institut für Kernphysik, Saupfercheckweg 1, D-69117 Heidelberg, Germany
}

(Dated: July 8, 2021)

\begin{abstract}
High-order harmonic generation (HHG) with relativistically strong laser pulses is considered employing electron ionization-recollisions from multiply charged ions in counterpropagating, linearly polarized attosecond pulse trains. The propagation of the harmonics through the medium and the scaling of HHG into the multi-kilo-electronvolt regime are investigated. We show that the phase mismatch caused by the free electron background can be compensated by an additional phase of the emitted harmonics specific to the considered setup which depends on the delay time between the pulse trains. This renders feasible the phase-matched emission of harmonics with photon energies of several tens of kilo-electronvolt from an underdense plasma.
\end{abstract}

PACS numbers: 42.65.Ky, 42.79.Nv

In the last decades, atomic high-order harmonic generation (HHG) in the non-relativistic regime [1] has been developed to a reliable source of coherent extreme ultraviolet (XUV) radiation and of attosecond pulses opening the door for attosecond time-resolved spectroscopy [2]. The further advancement of this technique into the hard x-ray domain would, in particular, allow for ultrafast coherent diffraction imaging of single particles, clusters and biomolecules with sub-ångström resolution, tracking the electron motion in atoms and even for the investigation of time-resolved dynamics of nuclear excitations. The large scale $\mathrm{x}$-ray free electron lasers are likely to fulfill this task partly but are limited to energies around $10 \mathrm{keV}$.

Is it possible to extend the table-top HHG sources into the hard x-ray domain? In principle, the harmonic photon energy can be increased by using stronger driving laser fields. The state-of-the-art technique allows to generate coherent $\mathrm{x}$-ray photons up to the $\mathrm{keV}$ energy range [3] and to produce short XUV pulses of less than 100 as [4] from non-relativistic HHG in an atomic gas medium. However, progress in this field appears to have reached a limit. Most importantly, the further increase of the driving field intensity transfers the interaction regime into the relativistic domain where the drift motion of the ionized electron in the laser field propagation direction prohibits the recollision and, consequently, suppresses HHG [5]. This happens at laser intensities above of $4 \times 10^{16} \mathrm{~W} / \mathrm{cm}^{2}$ at infrared wavelengths, corresponding to the HHG cutoff frequency of $\omega_{c} \approx 10 \mathrm{keV}$. This indicates the limit of non-relativistic HHG. The second point hindering HHG at high intensities is the phase-matching problem. In strong laser fields, outer-shell electrons are rapidly ionized and produce a large free electron background causing phase mismatch between the driving laser wave and the emitted x-rays.

Various methods for counteracting the relativistic drift have been proposed. However, no universal solution has been found, each method has its drawbacks. To suppress the drift, highly charged ions moving relativistically against the laser propagation direction [6] or a gas
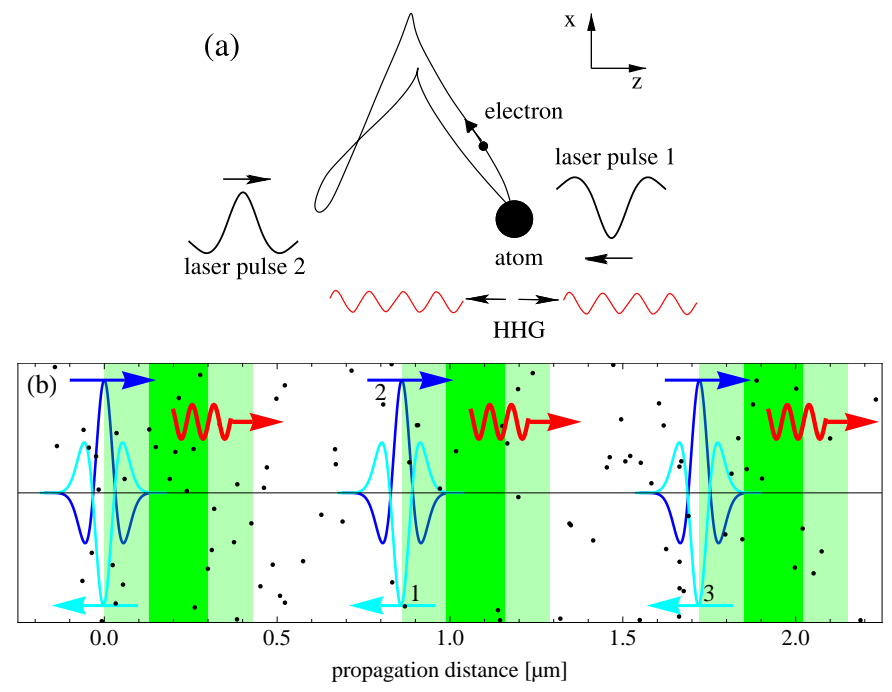

FIG. 1: (Color online) The HHG setup with counterpropagating APTs: (a) The trajectory of the rescattering electron; (b) The contribution of different parts of the medium to HHG. The harmonics emitted from the green (dark) area propagates along the red (wavy) arrow. The HHG from shaded area is damped. The two driving APTs are shown in different blue color shades. The arrows indicate the propagation direction.

of positronium atoms [7 can be used. Different combinations of laser fields also have been proposed for this purpose such as a tightly focused laser beam [8], two crossed laser beams with linear polarization [9, 10, or with equal-handed circular polarization [11. In the latter field configuration, the relativistic drift is eliminated, however, in this scheme the phase-matching is especially problematic to realize [12. We have shown in [13 15] that strong attosecond pulse trains (APTs) employed as a driving field for HHG can be very useful to suppress the relativistic drift. However, all these efforts have only addressed HHG of a single atom rather than coherent emission from a macroscopic gas target.

In this Letter, we investigate the feasibility of phase- 
matched harmonic emission from an underdense plasma of multiply charged ions for a relativistic HHG setup employing two counterpropagating APTs. We show that the HHG driven by counterpropagating APTs has an additional intrinsic phase depending on the time delay between the pulses as well as on the pulse intensity. This phase avails to compensate the phase mismatch between the driving laser field and the emitted harmonics due to the free electron background. The latter can be achieved by modulating the driving field intensity with a slowly decreasing envelope. We have performed a complete, quantitative analysis of the macroscopic yield of the relativistic HHG evidencing a small but detectable signal.

The applied setup for relativistic HHG is shown in Fig. 1(a). The driving fields are two counterpropagating APTs consisting of 100 as pulses with a peak intensity of the order of $10^{19} \mathrm{~W} / \mathrm{cm}^{2}$ and a spectral range of about $20 \mathrm{eV}$. Such pulses can be generated by employing the relativistic oscillating mirror of an overdense plasma surface in a strong laser field [16. The electron is liberated by one pulse, it is driven in the continuum and undergoes the relativistic drift. A moment later, the second pulse reaches the electron, it reverts the drift and realizes rescattering. The drift compensation is very efficient as one can deduce from Fig. 2 where the single-atom spectral emission rate in the present setup is compared with that in the dipole approximation (DA). The setup exhibits no significant drift any more. The rate for the applied setup is much higher than the one for a conventional laser field with the same cutoff. In the latter case, due to the drift, the rate would drop rapidly with increasing laser intensity.

We continue to estimate the macroscopic HHG yield of the given setup. The contributions of different parts of the medium to the harmonic emission are shown in Fig. 1 (b). Emission into the right direction is considered. HHG occurs only in small zones of the medium shown as dark areas in Fig. 1 (b). In this case, necessarily, recombination has to be arranged by a pulse propagating to the right. For instance, ions between pulse 1 and 3 in the sketch could have been ionized by pulse 1 , which triggered the process. In the next step, pulse 2 could arrange rescattering and HHG. This is possible only up to a certain point (dark area) since then pulse 2 and 3 meet and the process starts again. Contributions of atoms experiencing two pulses simultaneously (light shaded areas) are frustrated due to the chaotic trajectories of ionized electrons in this region [17. This limits the volume in longitudinal direction to about $1 / 3$ and the possible delays are within $1-2$ fs. After half a period, $1 / 3$ of the white area would emit into the right direction.

In the following, we specify our model. As HHG medium, an underdense plasma of $\mathrm{O}^{6+}$ ions (ionization potential $I_{p}=27.18$ a.u.) is used which is immediately formed when the first laser pulse of relativistic intensity is applied to a neutral atomic gas. This is because the outer shell electrons of an oxygen atom are almost instantaneously ionized due to a much smaller binding potential (0.5 a.u. -5.1 a.u.) other than the two remaining electrons in the closed $1 s$-shell. HHG is produced only by the tightly-bound inner electron having an ionization potential corresponding to the tunneling condition at relativistic intensities. The $\mathrm{O}^{6+}$ emission is slightly reduced by the depletion to $\mathrm{O}^{7+}$ whereas the $\mathrm{O}^{7+}$ emission is not phase-matched in the proposed phase-matching scheme. The driving laser pulses are plane waves numerically propagated in the relativistic free electron background using a Crank-Nicolson-algorithm. The density of the free electrons is assumed to be constant because the outer shell ionization time is small compared to the laser period. Absorption of the high-frequency HHG photons can be neglected because their energy is much higher than the largest atomic transition energy. In order to find the overall HHG yield, the photon spectral density $\frac{d N}{d \omega_{H}}$ in the far field is calculated (in atomic units) [18:

$$
\frac{d N}{d \omega_{H}}=\frac{c}{4 \pi^{2} \omega_{H}} R^{2} \int d \Omega^{\prime}\left|\tilde{\boldsymbol{E}}\left(\boldsymbol{n}^{\prime}, \omega_{H}\right)\right|^{2},
$$

where $\boldsymbol{n}^{\prime}$ is the emission direction, $R$ the radius at an observation point, $\omega_{H}$ the positive harmonic frequency. The spectral component of the electric field reads

$$
\tilde{\boldsymbol{E}}\left(\boldsymbol{n}^{\prime}, \omega_{H}\right)=i \frac{\omega_{H} \rho e^{-i \omega_{H} R / c}}{R c^{2}} \int d^{3} x_{a} \tilde{\boldsymbol{j}}_{a}\left(\boldsymbol{x}_{a}, \omega_{H}, \boldsymbol{n}^{\prime}\right),
$$

where $\rho$ is the density of the uniformly distributed ions, $\boldsymbol{j}_{a}\left(\boldsymbol{x}, t, \boldsymbol{x}_{a}\right)$ the current density at a space-time point $\boldsymbol{x}$ and $t$ of a single ion located at $\boldsymbol{x}_{a}$ and $\tilde{\boldsymbol{j}}_{a}\left(\boldsymbol{x}_{a}, \omega_{H}, \boldsymbol{n}^{\prime}\right)$

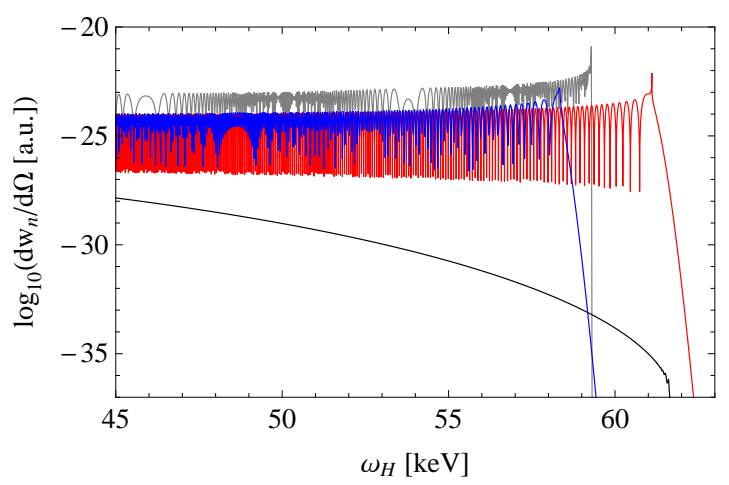

FIG. 2: (Color online) Single-atom HHG rates via [15] in laser propagation direction: (red, largest cutoff) for the discussed setup; the delay of pulses is $1.5 \mathrm{fs}$, the laser field strength $E_{0}=21$ a.u., and $I_{p}=27.18\left(\mathrm{O}^{6+}\right)$; (blue, lowest cutoff) for the same setup and parameters in DA including the mass shift; (black, bottom) for a conventional propagating laser field with $E_{0}=2.7$ a.u., $I_{p}=7.35$ a.u. (hydrogen-like ion), and (gray, top) the latter within the DA including the mass shift. The indicated parameters are chosen such that both the cutoffs and the average ADK-tunneling rates are the same for the two fully-relativistic curves. 
its Fourier transform which is calculated quantummechanically using the relativistic strong field approximation (SFA) 19. The emitted harmonics have the same polarization direction (along the $\mathrm{x}$-axis) as the incident laser field. The $\mathrm{x}$-component of the spectral electron current density at a single multiply charged ion in the SFA based on the Klein-Gordon equation reads 20]:

$$
\begin{array}{r}
\tilde{j_{a}}\left(\boldsymbol{x}_{a}, \omega_{H}, \boldsymbol{n}^{\prime}\right)=\frac{1}{4 c} \sqrt{\frac{\omega_{H}}{2 \pi}} \int d^{4} x \int d^{4} x^{\prime} \phi^{*}\left(\boldsymbol{x}-\boldsymbol{x}_{a}, t\right) \\
\times V_{H}(x) G\left(x, x^{\prime}\right) \kappa\left(t^{\prime}\right) V_{A I}\left(x^{\prime}\right) \phi\left(\boldsymbol{x}^{\prime}-\boldsymbol{x}_{a}, t^{\prime}\right),(3)
\end{array}
$$

where $\phi\left(\boldsymbol{x}-\boldsymbol{x}_{a}\right)$ is the wave function of the bound electron, $V_{H}(x)$ the interaction Hamiltonian of the electron with the harmonic field, $V_{A I}(x)$ the potential of the ionic core and $G\left(x, x^{\prime}\right)$ is the Green function describing the free electron evolution in the counterpropagating laser fields defined in [15. We included a tunneling correction factor $\kappa(t)=\sqrt{w_{A D K}(t) / w_{K}(t)}$ upgrading the Keldysh tunneling rate $w_{K}(t)$ comprised in the SFA to fit the ADKionization rate 21. As discussed, we only consider the relevant scenario of interaction where the electron moves in different counterpropagating pulses successively. Accordingly, in each stage of the excursion, we approximate the Green function by the Volkov Green function in a field of the appropriate single laser pulse (see [15). Eq. (3) is evaluated in the saddle point approximation.

Let us have a closer look at the phase difference of the harmonics emitted from different ions separated by a distance $\Delta z$ in the propagation direction:

$$
\Delta \varphi=\Delta \arg \tilde{j_{a}} \approx \Delta z\left(\frac{\omega_{H}}{c} \frac{\Delta v_{g}}{v_{g}}-\frac{\partial \varphi_{i}}{\partial z}\right)
$$

The first term describes the phase mismatch due to the free electron dispersion with $\Delta v_{g}=\omega_{p}^{2} / 2 \omega_{H}^{2}, \omega_{p}$ being the electron plasma frequency and $v_{g}$ the group velocity of the driving laser pulse, whereas the last term is the single-atom emission phase $\left(\varphi_{i}\right)$ depending on the laser field conditions. This intrinsic phase $\varphi_{i}$ is determined by the classical action of the electron trajectory recolliding with the specific harmonic energy and can be estimated as $\varphi_{i} \approx \tilde{U}_{p}\left(\boldsymbol{r}_{a}\right) \tau\left(\boldsymbol{r}_{a}\right)$, with an effective ponderomotive potential $\tilde{U}_{p}\left(\boldsymbol{r}_{a}\right)$ and electron excursion time $\tau\left(\boldsymbol{r}_{a}\right)$. Thus, $\varphi_{i}$ depends on the laser intensity as well as on the delay between the two pulses. The latter, being unique for this laser setup, mainly affects the electron excursion time $\tau\left(\boldsymbol{r}_{a}\right)$ and varies along the propagation direction. In order to achieve phase-matching, one can vary the laser intensity along the propagation direction to balance the intrinsic phase with the phase slip due to dispersion. The required intensity variation to have a constant complex phase $\arg \tilde{j_{a}}$ in the entire medium is calculated numerically and shown in Fig. 3 for the first interaction zone. It is optimized for the long trajectory of $50 \mathrm{keV}$ energy but could be accomplished for any energy value below. Note that only one of the short
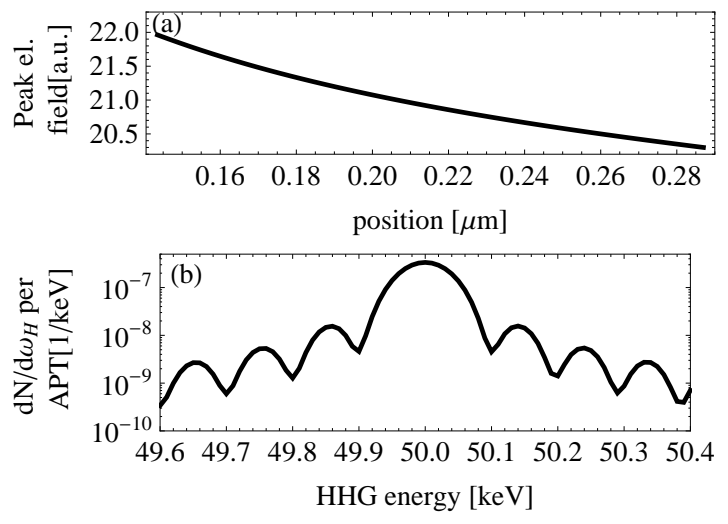

FIG. 3: (a) shows the optimized peak electric field variation in the first interaction zone. The excursion time increases to the right, (b) Emitted spectral photon number (spikes at integer multiples of $\omega$ do not appear due to local averaging) of the setup for the medium described in the text.

and long trajectories can be phase-matched since their classical actions are different. For the analytical description of the spatial variations of the laser field in the expression for $G\left(x, x^{\prime}\right)$, the eikonal-Volkov approximation is applied [22]. This is justified because the additional driving field causing the modulation perturbs the electron energy only slightly. The experimental realization of the phase-matched scheme could be achieved, e.g., with a modulated hollow core waveguide.

We employ a medium length as short as the spatial extent of the APT to minimize dispersion. In our simulation, each APT consists of 15 pulses with an APT duration of $40 \mathrm{fs}$. Our calculations show that in the case of longer APTs, the pulses in the train strongly spread due to dispersion and overlap, thus, violating the condition for the drift compensation. All pairs of pulses have almost the same coherent contribution to the overall yield. Since the pulses in different zones have experienced a different propagation length through the plasma, their shapes differ slightly. However, phase-matching still can be maintained by slightly adjusting the modulation profile, as long as the pulse shape still supports the recollision scheme. The phase-matching scheme imposes a strong demand on the jitter of the laser field $\Delta E / E$ : $\Delta U_{p} \tau \ll 1$ yields $\Delta E / E \ll\left(U_{p} \tau\right)^{-1} \sim 10^{-4}$. We choose a gas density of $\rho=10^{19} / \mathrm{cm}^{3}$ (ionized by the laser as described before), a diameter of $1 \mathrm{~mm}$ and a length of $12.5 \mu \mathrm{m}$ for the interaction volume. The emitted spectral photon number is shown in Fig. 3. An integral over the spectrum yields an emitted photon number of $2.5 \times 10^{-8}$ at $50 \mathrm{keV}$ per one collision of APTs which corresponds to a measureable signal of about 2 photons per day at $1 \mathrm{kHz}$ repetition rate. Note that the choice of the atomic species is rather flexible. Multielectron highly charged ions offer an enhanced recombination probability due to core polarization [23] but produce a larger electron background 


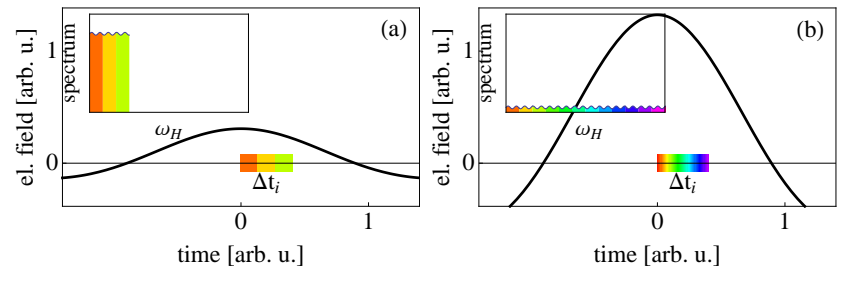

FIG. 4: Schematic illustration of the chirping factor $\partial \omega_{H} / \partial t_{i}$ : the harmonic bandwidth per unit ionization time. The insets show harmonic spectra for laser fields (black line) of either low (a) or high (b) intensity. The ionization time window $\Delta t_{i}$ resulting in HHG is marked on the time-axis, where the colors indicate the harmonic frequency originating from the particular ionization time. $\Delta t_{i}$ remains unchanged under the increase of intensity but the bandwidth of the contained harmonics increases from (a) to (b), consequently, decreasing the ionization propability per harmonic frequency expressed by an increase of the chirping factor.

that can be balanced by a lower gas density. The overall efficiency is maintained or could even be enhanced. The bandwidth of phase-matched HHG in this scheme is about $150 \mathrm{eV}$ near the cutoff and pulses with a duration of about 35 as can be produced.

The small magnitude of the harmonic signal compared to current XUV HHG yields can be explained by investigating the spectral HHG photon rate $\dot{N}_{n}$ for phase-matched emission [13, 21] of the harmonic order $n=\omega_{H} / \omega$ from a fixed volume

$$
\dot{N}_{n} \sim w_{i}\left(t_{i}\right)\left|\left\langle 0\left|V_{H}\right| \mathbf{p}\right\rangle\right|^{2}\left(v_{\perp}^{2} \tau^{2} \partial \omega_{H} / \partial t_{i}\right)^{-1} .
$$

Here $w_{i}\left(t_{i}\right)$ is the ionization rate with the ionization time $t_{i},\left\langle 0\left|V_{H}\right| \mathbf{p}\right\rangle$ the recombination amplitude and the last factor accounts for the dynamical properties of the wave packet. $v_{\perp}^{2} \tau^{2}$ expresses the transversal electron spreading with transversal spreading velocity $v_{\perp}, \tau$ the excursion time of the electron and $\partial \omega_{H} / \partial t_{i}$ is the so-called electron wave packet chirping factor discussed below.

We proceed by analyzing the scaling of $\dot{N}_{n}$ with increasing laser intensity at a harmonic energy near the respective cutoff provided that $w_{i}\left(t_{i}\right)$ is kept constant by an appropriate choice of $I_{p}$. The recombination amplitude decreases with increased electron energy favoring scattering rather than recombination. Its scaling depends on the shape of the ionic potential: $\left|\left\langle 0\left|V_{H}\right| \mathbf{p}\right\rangle_{C}\right|^{2} \sim I_{p}^{5 / 2} / \omega_{H}^{4}$ for a hydrogen-like ion and $\left|\left\langle 0\left|V_{H}\right| \mathbf{p}\right\rangle_{Z}\right|^{2} \sim \sqrt{I_{p}} / \omega_{H}^{2}$ for a zero-range potential with $I_{p} \ll \omega_{H}$ and $p^{2} \sim \omega_{H}$. Regarding the last term of Eq. (5), we follow 21] to find $v_{\perp}=\sqrt{E} / I_{p}^{1 / 4} \sim\left(\omega_{H} / I_{p}\right)^{1 / 4}$ and illustrate the chirping factor in Fig. 4. It describes that the bandwidth of the harmonics emitted from a fixed ionization time window rises with increasing laser intensity (i.e. the ionization probability per harmonic decreases) and can be estimated as $\partial \omega_{H} / \partial t_{i} \sim \omega_{H} / \Delta t_{i}$. Thus, the photon emission rate in a constant bandwidth for a zero-range potential scales as $\dot{N}_{n} \sim I_{p} / \omega_{H}^{3.5}$. A rough estimate for the scaling of $I_{p}$ at a constant ionization rate can be derived fixing the common tunneling exponent yielding $I_{p} \sim E^{2 / 3} \sim \omega_{H}^{1 / 3}$ and, consequently, $\dot{N}_{n} \sim 1 / \omega_{H}^{3.17}$. The decrease for a hydrogen-like potential is even more dramatic. Therefore, the HHG photon yield decreases with rising photon energy due to the decreased probabilities of ionization per harmonic and the reduced recombination cross section. Our analysis points out a possible future direction for optimization of HHG by means of increasing the ionization time window at a given harmonic bandwidth.

In conclusion, we have shown that the drift and phasematching do not restrict HHG to the non-relativistic regime. The proposed setup renders the relativistic regime of HHG in a multi-atom ensemble accessible.

* Corresponding author: k.hatsagortsyan@mpi-k.de

[1] P. B. Corkum, Phys. Rev. Lett. 711994 (1993); P. Agostini and L. F. DiMauro, Rep. Prog. Phys. 67, 813 (2004).

[2] F. Krausz and M. Ivanov, Rev. Mod. Phys. 81, 163 (2009).

[3] G. Sansone et al., Science 314, 443 (2006); T. Popmintchev et al., Proc. Nat. Acad. Sci. 106, 10516 (2009).

[4] E. Goulielmakis et al., Science 320, 1614 (2008).

[5] Y. I. Salamin et al., Phys. Reports 427, 41 (2006).

[6] G. Mocken, and C. H. Keitel, J. Phys. B 37, L275 (2004); C. C. Chirilă et al., Phys. Rev. Lett. 93, 243603 (2004).

[7] B. Henrich, K. Z. Hatsagortsyan and C.H. Keitel, Phys. Rev. Lett. 93, 013601 (2004).

[8] Q. Lin, S. Li, and W. Becker, Opt. Lett. 31, 2163 (2006).

[9] N. J. Kylstra et al., Phys. Rev. Lett. 85, 1835 (2000); V. D. Taranukhin, Laser Phys. 10, 330 (2000); M. Verschl, C. H. Keitel, Phys. Rev. ST AB 10, 024001 (2007).

[10] C. C. Chirilă et al., Phys. Rev. A 66, 063411 (2002).

[11] N. Milosevic, P. B. Corkum, and T. Brabec, Phys. Rev. Lett. 92, 013002 (2004).

[12] C. Liu et al., New J. Phys. 11, 105045 (2009).

[13] M. Klaiber, K. Z. Hatsagortsyan, and C. H. Keitel, Phys. Rev. A 74 051803(R) (2006); ibid. 75, 063413 (2007).

[14] M. Verschl and C. H. Keitel, J. Phys. B 40, F69 (2007).

[15] M. Klaiber et al., Opt. Lett. 33, 411 (2008); K. Z. Hatsagortsyan et al., J. Opt. Soc. Am. B 25, 93 (2008).

[16] Y. Nomura et al., Nature Phys. 5, 124 (2009).

[17] D. Bauer, P. Mulser, and W.-H. Steeb, Phys. Rev. Lett. 75, 4622 (1995).

[18] L. D. Landau and E. M. Lifshitz, The Classical Theory of Field (Pergamon, London, 1975).

[19] H. R. Reiss, Phys. Rev. A 42, 1476 (1990).

[20] D. B. Milošević, S. Hu, and W. Becker, Phys. Rev. A 63, 011403(R) (2000); Laser Phys. 12, 389 (2002).

[21] M. Y. Ivanov et al., Phys. Rev. A 54, 742 (1996).

[22] H. K. Avetissian et al., Phys. Rev. A 59, 549 (1999); O. Smirnova et al., Phys. Rev. A 77, 033407 (2008).

[23] A. Gordon et al., Phys. Rev. Lett. 96, 223902 (2006). 